\title{
Optical Sum Frequency Generation Image of Rice Grains
}

\author{
Hongyan $\mathrm{Li}^{1,2}$, Yoshihiro Miyauchi ${ }^{1,2}$, Nguyen Anh Tuan ${ }^{1,2}$, Goro Mizutani ${ }^{1,2 *}$, Mikio Koyano ${ }^{1}$ \\ ${ }^{1}$ Japan Advanced Institute of Science and Technology, Ishikawa, Japan; ${ }^{2}$ Japan Science and Technology Corporation, Core Research \\ for Evolutional Science and Technology, Tokyo, Japan. \\ Email: ${ }^{*}$ mizutani@jaist.ac.jp
}

Received January $4^{\text {th }}, 2012$; revised February $14^{\text {th }}, 2012$; accepted March $6^{\text {th }}, 2012$

\begin{abstract}
We observed optical sum frequency generation (SFG) images of cross-sections of glutinous rice grains, in order to test a possibility of the SFG microscopy as a tool for monitoring polysaccharide species in rice grains. The SFG response in the $\mathrm{CH}$ vibration range was the most intense in the crush cell layer at the edge of the endosperm adjacent to the embryo probably due to optical reflection and scattering effect by the rugged dielectric structure of the crush cell layer. The SFG spectra as a function of the infrared wavelength depended on the measurement position in the endosperm. The SFG results were compared with those by Raman and infrared spectroscopies for the same samples.
\end{abstract}

Keywords: Optical Sum Frequency Generation Microscopy; Oryzaglutinosa (Rice); Amylopectin; Starch; Crush Cell Layer; Endosperm

\section{Introduction}

Vibrational spectroscopy is a useful tool for characterizing biomaterials. The most frequently used are infrared (IR) absorption and Raman spectroscopies, and they give different information from each other because of their different symmetry selection rules [1-4]. Second-order nonlinear optical response has been also proposed as a viable tool for analyzing biological samples. By optical second harmonic generation ( $\mathrm{SHG}$ ) microscopy one gets images of non-centrosymmetric parts in biological samples without their pretreatment and damage [5,6]. By optical sum frequency generation (SFG) spectroscopy one can generally distinguish between different biomaterial species.

In this study we analyze SFG from the endosperm of glutinous rice grains in the $\mathrm{CH}$ vibrational range in order to check if the SFG can be a tool for observing the polysaccharide distribution in them. In a SFG phenomenon two light beams with different photon energies $\hbar \omega_{1}$ and $\hbar \omega_{2}$ irradiate the medium and another beam with their sum photon energy $\hbar \omega_{1}+\hbar \omega_{2}$ is emitted. SFG occurs in a medium without inversion symmetry and it detects chirality in the bulk medium. By using the resonance characteristics of one IR beam with photon energy $\hbar \omega_{2}$ to molecular vibrations in the medium, one can distinguish between different molecular species. An optical sum frequency microscopic image is obtained by mapping the distribution of the molecular vibration.

The demonstration of the SFG microscopy was per-

"Corresponding author. formed for the first time by Flörsheimer and his coworkers for Langmuir-Blodget films in 1999 [7]. Only a few number of SFG image observations of living organisms have been done afterwards. As for a botanical system, Miyauchi and his coworkers were the first to carry out the SFG microscopy [8]. Their sample was a water plant Charafibrosa. Inoue and his coworkers reported an SFG microscopy observation of an onion root cell [9]. In order to develop the application range of this microscopy, we further attempted to monitor the polysaccharide distribution in the cross-sections of rice grains in this study.

Our another trigger for observing rice grains was the fact that we observed intense SHG and SFG from starch from living Chara in our previous studies [8,10]. The structure of $\alpha$-D-glucopyranose constituting the polysaccharides in rice has chirality and lacks inversion symmetry. In the starch crystalline domains, amylopectin chains have orderly structures and have intense SHG and SFG response [8]. Starch consists of amylopectin and amylose. The starch of non-glutinous rice consists of $80 \%$ amylopectin and $20 \%$ amylose, while that of the glutinous rice is almost $100 \%$ amylopectin [11]. Miyauchi and his coworkers proposed that SFG in Chara originates from amylopectin [8]. Zhuo and his coworkers [6] proved SHG occurs in amylopectin in rice. We chose glutinous rice as our sample, since stronger SFG is expected from it than from non-glutinous rice.

Monitoring the distribution of polysaccharides in rice grains during their growth should be a very important research topic in order to improve the breed of rice. After 
the rice flower gets fertilized, the endosperm nucleus in the ovary grows into a full endosperm and then the rice ear grows. Enzymes make small polysaccharide clusters grow into groups of amylopectin and amylose and then starch granules. Here it is a hot research topic to control the functions of the relevant enzymes using biotechnology and produce various kinds of useful starches $[12,13]$. Understanding the growth or digestion process of starch in the growing or germinating ovary can contribute to the controlling technology of the starch structure and the breed improvement of rice against cold or bad environment. SFG response is sensitive to the higher order structure of polysaccharide $[8,9]$, so the attempt to analyze rice grains by SFG microscopy can be a good step to contribute to rice science and technology.

The purpose of this study is to check the capability of the SFG microscopy of observing the distribution of density and compositions of polysaccharides in the cross sections of glutinous rice grains. From this viewpoint we have also performed Raman and IR spectroscopies of the same samples, in order to check the difference between the information provided by SFG and these other vibrational spectroscopies. As a future potential topic of research we are also interested in studying the transformation of starch in their growth and digestion.

\section{Materials and Methods}

Figure 1 shows the setup of the optical sum frequency generation confocal microscope [14]. As a visible light source of wavelength $532 \mathrm{~nm}$ (photon energy $2.33 \mathrm{eV}$ ) we used a doubled frequency output from a mode-locked cavity-dumped $\mathrm{Nd}^{3+}$ : YAG laser operating at a repetition rate of $10 \mathrm{~Hz}$. As a light source of wavelength-tunable IR light we used an output with wavelength $\sim 3.4 \mu \mathrm{m}$ (wave number $\sim 2950 \mathrm{~cm}^{-1}$ ) from an optical parametric generator and amplifier system (OPG/OPA) driven by the same $\mathrm{Nd}^{3+}$ : YAG laser. In the following we use $\mathrm{eV}$ and $\mathrm{cm}^{-1}$ units to indicate the photon energy of the visible light and the wave number of the IR light, respectively. The band width of the IR light was narrower than $6 \mathrm{~cm}^{-1}$. The visible light of photon energy $2.33 \mathrm{eV}$ passed through a

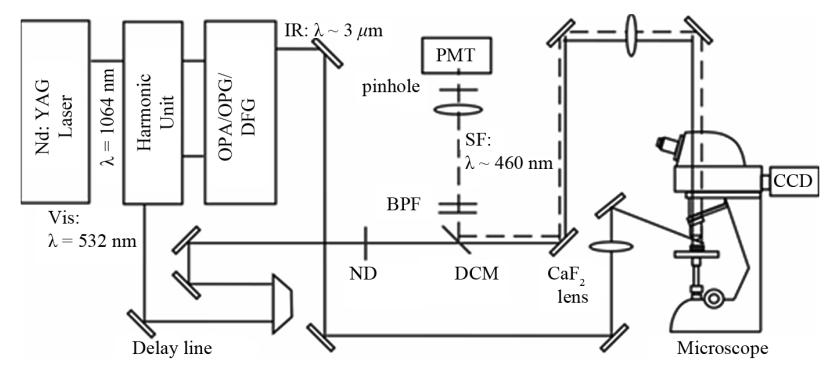

Figure 1. Optical setup for the sum frequency generation (SFG) confocal microscopy. dichroic mirror (DCM: Semrock, FF506-Di02) with transmission of $\sim 95 \%$. It was then focused on the sample with $20 \mathrm{X}$ objective lens (numerical aperture $=0.45$ ) in a commercial microscope (Nikon Eclipse: LV100D) in the imaging optics. The spot size of the $2.33 \mathrm{eV}$ beam on the rice sample was $\sim 1 \mu \mathrm{m}$. The IR beam was focused on the sample with a $\mathrm{CaF}_{2}$ lens with a focal length of $200 \mathrm{~mm}$ with the spot sizes on the sample of $50-100 \mu \mathrm{m}$. The pulse energies of the visible and IR beams at the sample surface were $<1$ and $30 \mu \mathrm{J} /$ pulse, and their incident angles were $0^{\circ}$ and $50^{\circ}$, respectively. The illumination of the sample by the visible and the IR beams were adjusted to be spatially and temporarily coincident with each other by using mirrors and a delay line. We used a red diode laser beam propagating collinearly with the IR laser beam to optimize the overlap of the visible and IR beam spots on the sample with the naked eyes.

The SFG light from the sample was collected by the objective lens and a tube lens of focal length $f=200 \mathrm{~mm}$ in the microscope optics. It was made into a parallel beam with a lens of $\mathrm{f}=200 \mathrm{~mm}$ and propagated back on the same optical path as the incident beam. It was then reflected by the DCM, passed through band pass filters, a lens with focal length $\mathrm{f}=100 \mathrm{~mm}$, and a pinhole with diameter 1 or $2 \mathrm{~mm}$, and finally detected by a photomultiplier. The spatial resolution of the system was $2 \mu \mathrm{m}$. The IR pulse energies were monitored by a photodiode and was used to normalize the SFG intensity.

The scanning range of the $\mathrm{x}-\mathrm{y}$ sample stage was $100 \times$ $100 \mu \mathrm{m}^{2}$. The moving steps of the piezo stages were 1 $\mu \mathrm{m}$ in all the directions. Manual x-y stages were put under the piezo stages in order to extend the scanning range. All the measurements were performed in air at room temperature of $24^{\circ} \mathrm{C}$.

For FT-IR measurement of the rice endosperm we used a Perkin-Elmer FT-IR microscope system AutoI$\mathrm{MAGE}^{\mathrm{TM}} /$ Spectrum $^{\mathrm{TM}}$ 100. The measurement was performed in the attenuated total reflection (ATR) configuration. The aperture size was $50 \times 50 \mu \mathrm{m}^{2}$ on the sample, the spectral range was from 750 to $4000 \mathrm{~cm}^{-1}$, and the energy resolution was $2 \mathrm{~cm}^{-1}$. The distortion of the spectral shape due to the ATR configuration was not corrected for.

Room temperature Raman scattering measurements were performed in 180 degree backscattering geometry by using a Horiba-Jobin Yvon Ramanor T64000 triple monochromator with $1800 \mathrm{gr} / \mathrm{mm}$ grating equipped with a CCD detector. The excitation source was the $514.5 \mathrm{~nm}$ emission from an $\mathrm{Ar}^{+}$laser with the power of $1 \mathrm{~mW}$. The spatial resolution of the measurement was $1 \mu \mathrm{m}$. The Stokes shift range was from 750 to $3800 \mathrm{~cm}^{-1}$. The energy resolution was $1.5 \mathrm{~cm}^{-1}$ between $750 \mathrm{~cm}^{-1}$ and 2008 $\mathrm{cm}^{-1}$, and $0.5 \mathrm{~cm}^{-1}$ between $2008 \mathrm{~cm}^{-1}$ and $3800 \mathrm{~cm}^{-1}$.

The kernels of the glutinous rice Oryzaglutinosa cv. 
Shintaishomochi were harvested in the year 2009 and were kept in a dry box at room temperature. By carefully cutting the kernels with a thin blade cutter, flat cross section specimens were prepared.

\section{Results and Discussion}

The photograph in Figure 2 shows a cross section of a grain of Oryzaglutinosa cv. Shintaishomochi. We see a wide milky-colored area called an endosperm to the left side and a smaller brown area called an embryo to the right side. A plumule and a radical are seen in the embryo. A brighter white zone is seen on the boundary between the endosperm and the embryo. It is a part of the endosperm and is called a crush cell layer. In this crush cell layer the structure is porous, and water tends to soak and spread efficiently and the neighboring starch is digested through hydrolysis [15]. During germination the starch is digested in the endosperm and supplies the embryo with nutrition through the crush cell layer.

Figure 3(a) shows a magnified photograph of the area in the black rectangular frame in Figure 2. We see expanded images of the endosperm to the left and the embryo to the right. In the center we see the crush cell layer as a bright curved zone of width about $50 \mu \mathrm{m}$. Figure 3(b) is a SFG image of the same area as Figure 3(a) with the IR wave number of $2924 \mathrm{~cm}^{-1}$. In Figures 3(a) and (b) the center areas emit the strongest signal. Hence SFG occurs most strongly in the crush cell layer at the edge of the endosperm. SFG from the endosperm to the left in Figure 3(b) is stronger than that from the embryo to the right. From the SFG intensity profile integrated in the vertical direction in the yellow frames drawn in Figure 3(b), the average SFG intensity in the area I is calculated as 3.5 times stronger than that in the area II.

The amyloplast containing starch granules in the endosperm is digested by enzymes, broken into pieces, and forms crush cell layer at the boundary between the endosperm and the embryo [11]. This crush cell layer scatters light strongly and is seen as the most white part in the cross section of the rice grain as seen in Figure 2 and Figure 3(a). SFG is also the strongest in this crush cell layer as seen in Figure 3(b). This correlation indicates

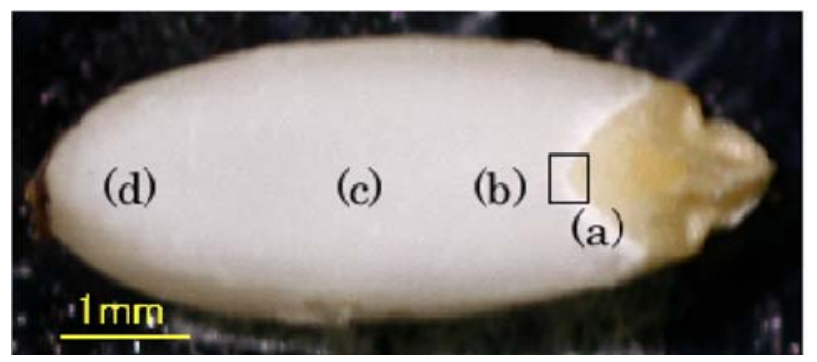

Figure 2. Cross-section photograph of a grain of Oryzaglutinosa cv. Shintaishomochi.

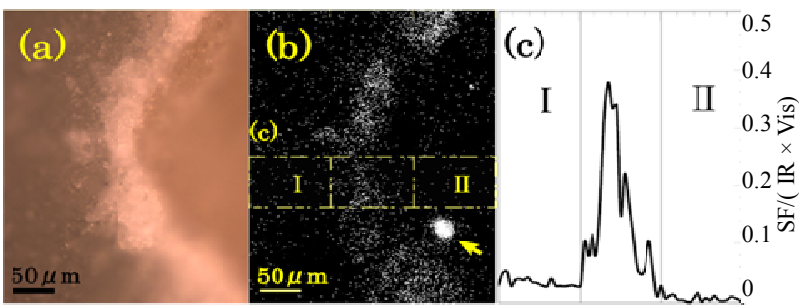

Figure 3. (a) Visible and (b) SFG images of a cross section of a glutinous rice grain around the crush cell layer between the endosperm (to the left) and the embryo (to the right). White dots represent light signals. The IR wave number for SFG was $2924 \mathrm{~cm}^{-1}$. The big spot indicated by an arrow in (b) is from an unknown particle unrelated to the glutinous rice grain. The measurement time of the SFG image was $204 \mathrm{~min}$; (c) SFG intensity profile as a function of the horizontal position in the area indicated by the yellow frames in (b).

that the intense SFG in the crush cell layer is due to the intense random reflection of the fundamental light by the rugged dielectric structure in the crush cell layer. Strong bulk SFG light is generated by the reflected fundamental light, travels through the starch granules towards the objective lens, and is detected. A similar enhancement of reflected bulk SHG intensity by a discontinuous refractive index structure is reported for a thin GaAs crystalline slab [16].

In order to identify the material emitting the SFG light in the crush cell layer, we measured SFG spectrum in the $\mathrm{CH}$ and $\mathrm{CH}_{2}$ vibrational region of the crush cell layer as shown in Figure 4(a). We also measured SFG spectra at several positions in the endosperm as shown in Figure 4(b) to (d). The sampling positions are shown in Figures 2, and the sampling size was approximately $1 \mu \mathrm{m}$. We call the four sampling areas as (a) the crush cell layer (b) the endosperm near the crush cell layer (c) the center of the endosperm, and (d) the outer edge of the endosperm. The IR frequency was scanned in the $\mathrm{CH}$ stretching vibration range from 2750 to $3150 \mathrm{~cm}^{-1}$. Since $\mathrm{SFG}$ spectra had sample dependence, we performed measurements for 5 samples and have obtained the average spectra in Figure 4.

Each of Figures 4(a) to (d) has two peaks at around $2905 \mathrm{~cm}^{-1}$ and around $2970 \mathrm{~cm}^{-1}$, and one dip at 2950 $\mathrm{cm}^{-1}$. In Figures 4(a) and (b) the two peaks are relatively strong compared to the background continuum signal, while in Figures 4(c) and (d) the two peaks have comparable intensity with the background. The SFG peak intensity at $2905 \mathrm{~cm}^{-1}$ relative to that of $2970 \mathrm{~cm}^{-1}$ in Figure 4(a) is larger than that in (b). The SFG peak intensity at $2905 \mathrm{~cm}^{-1}$ in Figure 4(c) looks large, but this is rather due to the slope of the background. These results mean that either the molecular species or orientation of molecules in the endosperm are different between the crush cell layer and the other parts in the endosperm. 


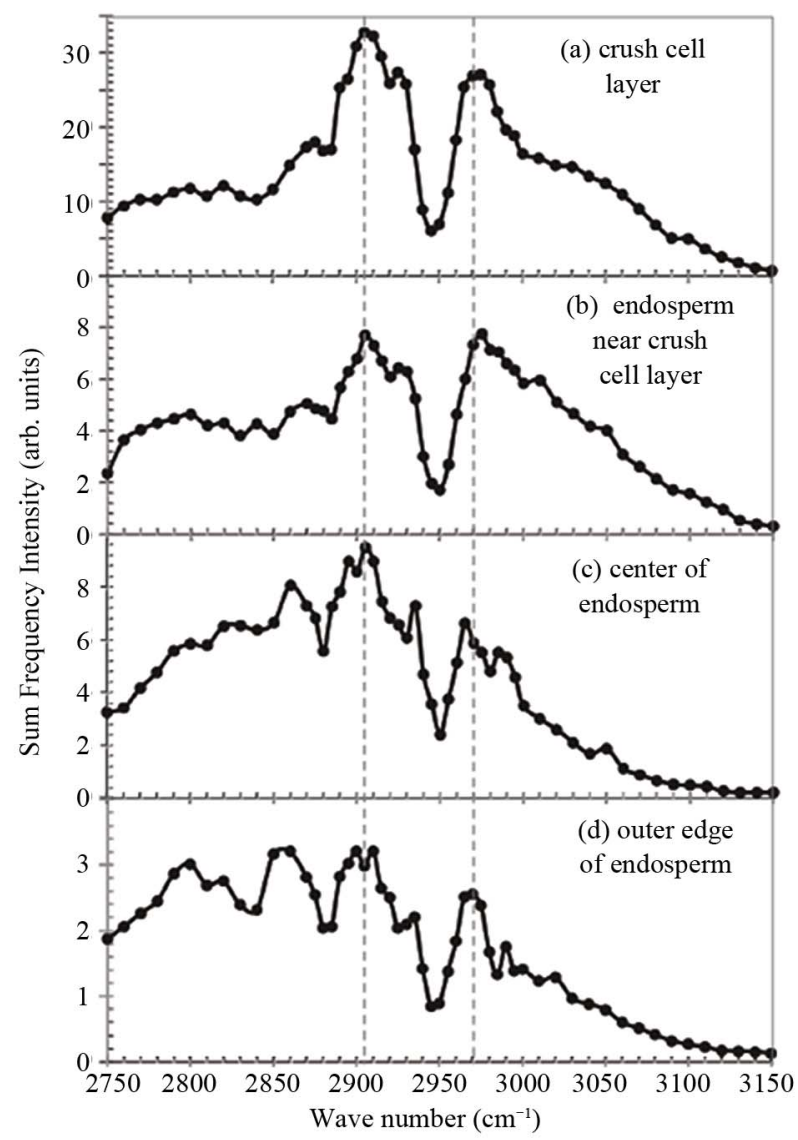

Figure 4. SFG spectra measured at four positions named (a) to (d) in Figure 2 in the endosperm of the glutinous rice grain.

It is well-known that the endosperm contains a lot of starch. Starch consists of amylopectin and amylose. In glutinous rice the starch contains nearly $100 \%$ amylopectin [11]. In the endosperm of our samples the existence of the amylopectin was confirmed by iodo-starch reaction using iodine-aqueous solution. In order to interpret the spectra in Figure 4, we show the SFG spectrum of amylopectin in Figure 5(a). The spectra in Figure 4 and that in Figure 5(a) resemble each other very much except for detailed differences. Therefore, amylopectin is the main ingredient in the material showing the SFG spectrum in Figure 4. Here we must note that SFG microscope is insensitive to amylose [8] and we have to use other methods such as IR or Raman microcopy to detect amylose.

As we already mentioned above, the peak at $2905 \mathrm{~cm}^{-1}$ in Figure 4(a) is seen to be a little more prominent than in (b), (c), and (d), if the background slopes are considered. This tendency is confirmed again later in Figure 6. One tentative interpretation of the feature of this SFG spectrum is obtained from a weighted average of the spectra of amylopectin in Figure 5(a) and glucose in (b) in the ratio of 15:100 as shown in Figure 5(c). Figure 5(c) reproduces the feature of Figure 4(a) qualitatively.

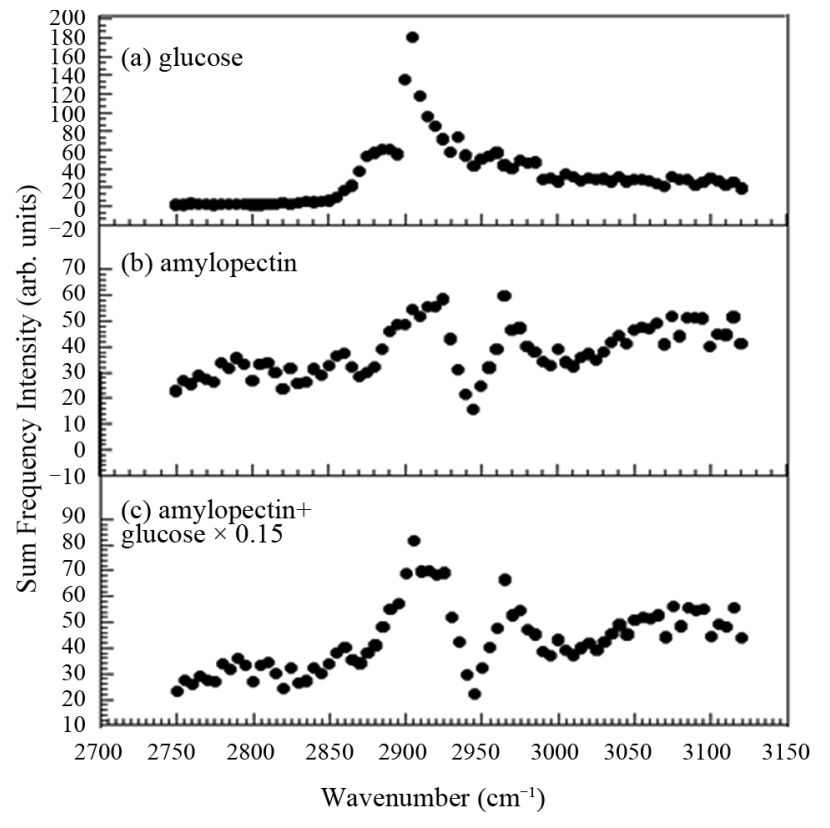

Figure 5. Optical sum frequency generation intensity of (a) amylopectin and (b) glucose and (c) their weighted average in the ratio of 15:100.

In rice grains amylopectin is supposed to be digested into glucose by amylase for germination and this is expected to occur very much at the crush cell layer between the endosperm and the embryo. Thus relatively larger abundance of glucose at the crush cell layer is plausible. However, since there are many other candidate species of saccharides to be considered, more reliable interpretation is to be obtained after contribution of all the possible saccharide species are taken into account in future analyses.

We have stated above that the intense white image of the crush cell layer between the endosperm and the embryo in the visible photograph in Figure 3(a) is due to the random back reflection of the incident light by structures in the crush cell layer. This means that the intense SFG signal in the crush cell layer does not necessarily indicate the relative abundance of amylopectin in the boundary region. In order to find a quantitative distribution of polysaccharide species in the crush cell layer, we need to obtain correction factors due to the optical reflection effects by monitoring optical signals from some marker material doped in samples.

Nevertheless, we may be able to have information on the relative abundance of materials from SFG intensity profiles. Figure 6 shows the SFG intensity profile for the IR wavenumber $2750 \mathrm{~cm}^{-1}$ and $2905 \mathrm{~cm}^{-1}$ as a function of the position on a straight line perpendicular to the crush cell layer between the endosperm and the embryo of one rice grain. The total areas of the two curves are normalized to the same value. For the two curves in Figure 6 we can regard the linear optical correction factors 


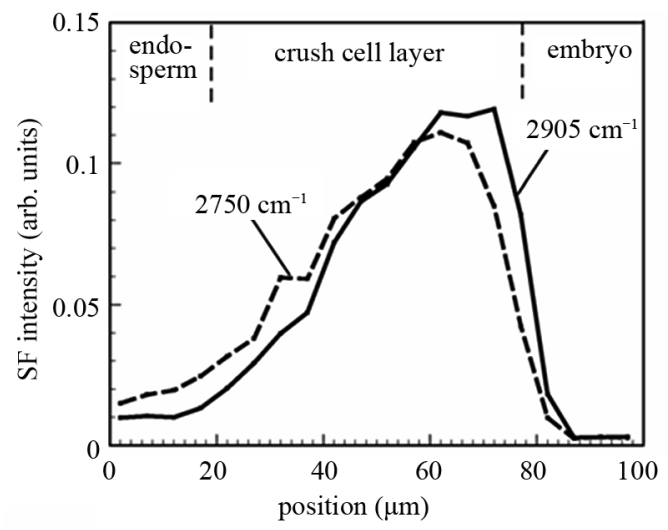

Figure 6. SFG signal intensity profile as a function of the position along a line perpendicular to the boundary between the endosperm and the embryo. The integrated areas of the two curves are normalized to the same value.

as similar to each other because all the experimental parameters except the IR wave number are common. Thus the difference of the two curve shapes reflects the difference of material distribution. In Figure 6 we can see that the SFG intensity at $2905 \mathrm{~cm}^{-1}$ is stronger in the part in the crush cell layer adjacent to the embryo. This fact suggests that the material generated by the digestion of amylopectin, presumably glucose, is accumulated in this area. Here we note that there is large sample and position dependence in the SFG intensity profile in the crush cell layers as we see it between Figures 3(c) and 6. Hence, the same position in the same rice grain must be observed, in order to compare the SFG intensity profiles for different IR wave numbers.

In order to check the difference between the information provided by SFG and by other vibrational spectroscopies, we have performed Raman and IR spectroscopies of the same samples. Figure 7(a) shows Raman scattering spectra of the endosperm and the crush cell layers on the cross section of a grain of Oryzaglutinosa cv. Shintaishomochi. The two areas show essentially the same Raman spectra in the whole spectral range. The broad background is a luminescence from the sample. We see peaks at $2910 \mathrm{~cm}^{-1}$, and there are many peaks in the finger print region between $800 \mathrm{~cm}^{-1}$ and $1500 \mathrm{~cm}^{-1}$. Figure 7(b) shows Fourier-transform infrared absorption (FT-IR) spectra of the two areas. The spectra do not show difference in the area near $3000 \mathrm{~cm}^{-1}$ but there are some differences in the range below $1800 \mathrm{~cm}^{-1}$.

The Raman and IR spectra of starch have been reported by Kizil et al. [17]. Our Raman spectrum in Figure 7(a) is consistent with their observation. Thus all the peaks seen in Figure 10(a) originate from starch in the sample. According to Kizil et al. many FT-IR peaks in Figure 7(b) are assigned to starch. The peak at 1654 $\mathrm{cm}^{-1}$ observed at the endosperm is water adsorbed to the amorphous regions of starch. We see Amide I, II, and III

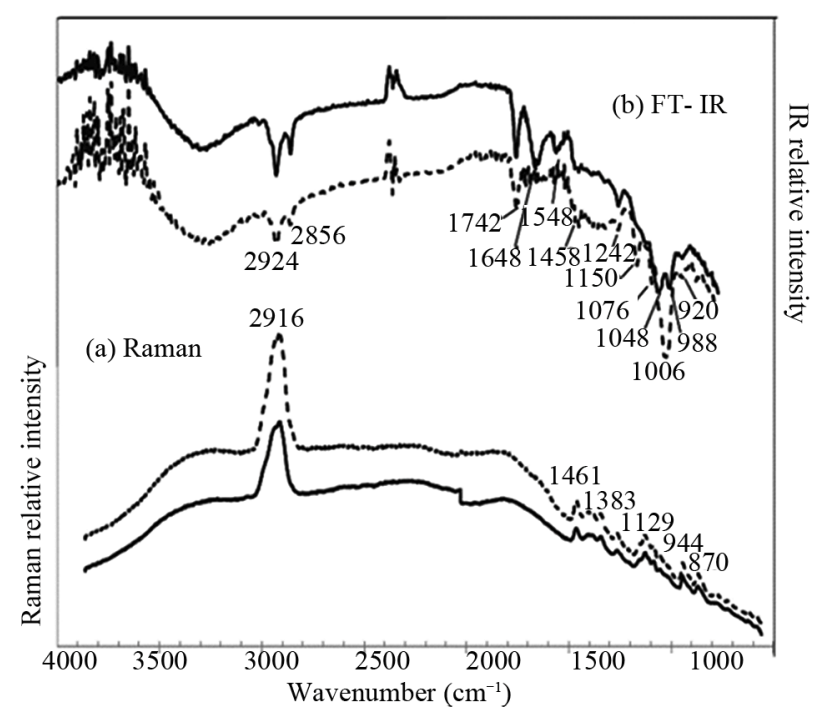

Figure 7. Raman scattering and infrared absorption spectra of the endosperm (solid curves) and the crush cell layer (dotted curves) on the cross section of a glutinous rice grain.

peaks at $1648 \mathrm{~cm}^{-1}, 1548 \mathrm{~cm}^{-1}$, and $1242 \mathrm{~cm}^{-1}$, respectively, in the ATR-IR spectra of the endosperm. In the crush cell layer we see Amide III peak. These Amide peaks should come from the protein ingredient in the rice grain.

If we limit ourselves to the frequency range near 2900 $\mathrm{cm}^{-1}$, the SFG spectra have more fine structures and thus are more sensitive to the difference of saccharide species in the endosperm of the glutinous rice than Raman or IR spectroscopy. In the IR spectrum the peak at $2920 \mathrm{~cm}^{-1}$ can be assigned to both starch and protein. The IR spectroscopy is sensitive to starch rather in the range from $900 \mathrm{~cm}^{-1}$ to $1200 \mathrm{~cm}^{-1}$. The IR spectra in the range from $1200 \mathrm{~cm}^{-1}$ to $1700 \mathrm{~cm}^{-1}$ can give information of protein. SFG and IR spectra together are expected to give the most useful information of the molecular species in rice grains.

\section{Conclusion}

We have observed images of cross sections of glutinous rice grain without germination with a confocal optical sum frequency generation microscope with a spatial resolution of $2 \mu \mathrm{m}$. SFG occurred most strongly in the crush cell layer at the edge of the endosperm. SFG from the endosperm was stronger than that from the embryo by 3.5 times. The results demonstrated that either the molecular species or the orientation of molecules in the endosperm are different between the crush cell layer and the other parts in the endosperm.

\section{Acknowledgements}

We would like to thank Prof. Nakamura and Prof. Fujita of Akita Prefectural University, and Prof. Hasegawa of 
Shiga Prefectural University for their valuable advice. We would like to thank Mr. Murai for providing us with the rice grains. We would also like to thank Prof. Kaneko and Mr. Yazaki of Japan Advanced Institute of Science and Technology for their technical support in the FT-IR measurement.

\section{REFERENCES}

[1] P. J. Treado and M. D. Morris, "Infrared and Raman Spectroscopic Imaging," Applied Spectroscopy Reviews, Vol. 29, No. 1, 1994, pp. 1-38. doi:10.1080/05704929408000896

[2] V. F. Kalasinsky, "Biomedical Applications of Infrared and Raman Microscopy," Applied Spectroscopy Reviews, Vol. 31, No. 3, 1996, pp. 193-249. doi:10.1080/05704929608000570

[3] J. X. Cheng, Y. K. Jia, G. Zheng and X. S. Xie, "LaserScanning Coherent Anti-Stokes Raman Scattering Microscopy and Applications of Cell Biology," Biophysical Journal, Vol. 83, No. 1, 2002, pp. 502-509. doi:10.1016/S0006-3495(02)75186-2

[4] S. W. Ellepola, S. M. Choi, D. L. Phillips and C. Y. Ma, "Raman Spectroscopic Study of Rice Globulin," Journal of Cereal Science, Vol. 43, No. 1, 2006, pp. 85-93. doi:10.1016/j.jcs.2005.06.006

[5] Y. R. Shen, "The Principles of Nonlinear Optics," John Wiley \& Sons, New York, 1984.

[6] Z. Y. Zhuo, C. S. Liao, C. H. Huang, J. Y. Yu, Y. Y. Tzeng, W. Lo, C. Y. Dong, H. C. Chui, Y. C. Huang, H. M. Lai and S. W. Chu, "Second Harmonic Generation Imaging-A New Method for Unraveling Molecular Information of Starch," Journal of Structural Biology, Vol. 171, No. 1, 2010, pp. 88-94. doi:10.1016/j.jsb.2010.02.020

[7] M. Flörsheimer, C. Brillert and H. Fuchs, "Chemical Imaging of Interfaces by Sum-Frequency Generation," Materials Science and Engineering C, Vol. 8-9, 1999, pp. 335-341. doi:10.1016/S0928-4931(99)00047-8

[8] Y. Miyauchi, H. Sano and G. Mizutani, "Selective Observation of Starch in a Water Plant Using Optical SumFrequency Microscopy," Journal of Optical Society of America A, Vol. 23, No. 7, 2006, pp. 1687-1690.

\section{doi:10.1364/JOSAA.23.001687}

[9] K. Inoue, M. Fujii and M. Sakai, "Development of a NonScanning Vibrational Sum-Frequency Generation Detected Infrared Super-Resolution Microscope and Its Application to Biological Cells," Applied Spectroscopy, Vol. 64, No. 3, 2010, pp. 275-281. doi:10.1366/000370210790918481

[10] G. Mizutani, Y. Sonoda, H. Sano, M. Sakamoto, T. Takahashi and S. Ushioda, "Detection of Starch Granules in a Living Plant by Optical Second Harmonic Microscopy," Journal of Luminescence, Vol. 87-89, 2000, pp. 824-826. doi:10.1016/S0022-2313(99)00428-7

[11] K. Hoshikawa, "Germination," In: T. Matsuo, et al., Eds., Science of the Rice Plant, Vol. 1, Morphology, Food and Agriculture Policy Research Center, Tokyo, 1993, pp. 7679.

[12] N. Fujita, M. Yoshida, N. Asakura, T. Ohdan, A. Miyao, H. Hirochika and Y. Nakamura, "Function and Characterization of Starch Synthase I Using Mutants in Rice," Plant Physiology, Vol. 140, No. 3, 2006, pp. 1070-1084. doi:10.1104/pp.105.071845

[13] Y. Nakamura, "Toward a Better Understanding of the Metabolic System for Amylopectin Biosynthesis in Plants: Rice Endosperm as a Model Tissue," Plant Cell Physiology, Vol. 43, No. 7, 2002, pp. 718-725. doi:10.1093/pcp/pcf091

[14] K. Locharoenrat, H. Sano and G. Mizutani, "Demonstration of Confocal Sum Frequency Microscopy," Physica Status Solidi (c), Vol. 6, 2009, pp. 304-306.

[15] S. Takahashi, "Physiology of Seed Germination," In: T. Matsuo, et al., Eds., Science of the Rice Plant, Vol. 2, Physiology, Food and Agriculture Policy Research Center, Tokyo, 1993, pp. 35-40.

[16] H. Sano, T. Shimizu, G. Mizutani and S. Ushioda, "Images of Cleaved GaAs(110) Surfaces Observed with a Reflection Optical Second Harmonic Microscope," Journal of Applied Physics, Vol. 87, No. 4, 2000, pp. 16141619. doi:10.1063/1.372068

[17] R. Kizil, J. Irudayaraj and K. Seetharaman, "Characterization of Irradiated Starches by Using FT-Raman and FTIR Spectroscopy," Journal of Agricultural Food Chemistry, Vol. 50, No. 14, 2002, pp. 3912-3918. doi:10.1021/jf011652p 